\title{
SPECTRAL SHIFT FUNCTION, AMAZING AND MULTIFACETED
}

\author{
M. Sh. Birman and A. B. Pushnitski
}

Several formula representations for the I. M. Lifshits - M. G. Kreı̆n spectral shift function (SSF) are discussed and intercompared. It is pointed out that the equivalence of these representations is not apparent, and different properties of the SSF are revealed by different formulas. The presentation is informal and contains no proofs.

To the memory of the great mathematician Mark Grigor'evich Kreı̆n

\section{Introduction}

The suggested notes discuss the theory of the spectral shift function (SSF), an important object in the spectral theory of perturbations, which covers both discrete and continuous spectrum. This notion was first singled out by the outstanding theoretical physicist I. M. Lifshits in his investigations in the solid state theory. In the paper [1] he presented his considerations "specially for mathematicians". There he kept within the bounds of the finite rank perturbations and limited himself to the formal aspect of the matter. The SSF was brought into mathematical use in M. G. Kreı̆n's famous paper [2], where the precise statement of the problem was given, the "proper" class of perturbations (trace-class operators) was found, and the explicit representation of the SSF in terms of the perturbation determinant was obtained. Since then, the "presence" of the SSF was discovered in a number of problems of both abstract-operator and quite concrete character. M. G. Kreŭn himself devoted several more papers to the SSF though this subject matter cannot be characterized as a main direction of his creative work. However, the first of the authors remembers quite well how thorough and meticulous was M. G. Kreı̆ in choosing the name for the new object (in the papers [1], [2] the term "SSF" does not occur).

The work of M. G. Kreın on the SSF has been described in detail in a recent survey

[9]; the further development of the SSF theory has also been discussed there. The aim of the present paper is somewhat different. It is to describe and to correlate several formula representations for the SSF. There are at least four of such representations found by now. 
Each of them adequately depicts some features of the SSF, but only considered together they lead to a correct understanding of this particular object.

Below we discuss only the initial version of the theory, when the perturbation of a selfadjoint operator is a trace-class selfadjoint operator. However, one should bear in mind that in applications a perturbation is usually "relatively trace-class" in an appropriate sense.

No proofs are presented in the paper. The technical details can be found in the survey [9] and in the book [10]. Section 6 discusses a representation for the SSF in terms of the counting functions of the spectrum of some family of compact operators. It has been found by the second of the authors and appears here for the first time; a detailed exposition will be published in [19].

Finally, we note that along with [2], M. G. Kreun has devoted his papers [3]-[8] to the SSF. The influence of M. G. Kreı̆n's papers turned out to be lasting and enduring.

Basic notation. Below, $\mathcal{H}$ is the basic (separable) Hilbert space, $\|\cdot\|$ is the norm and $(\cdot, \cdot)$ the inner product in $\mathcal{H}$. For a linear operator $A$ acting in $\mathcal{H}$, Ran $A$ denotes its range, and $\operatorname{rank} A:=\operatorname{dim} \operatorname{Ran} A ; A^{*}$ is the adjoint operator; $\sigma(A)$ is the spectrum of $A$, $\rho(A)=\mathbf{C} \backslash \sigma(A)$ is the resolvent set; and $R_{z}(A)=(A-z I)^{-1}, z \in \rho(A)$, is the resolvent of $A$. For $A=A^{*}$ denote by $E_{A}(\cdot)$ the spectral measure for $A$.

Let $\mathbf{S}_{\infty}(\mathcal{H})$ be the class of compact operators in $\mathcal{H}$. If $T \in \mathbf{S}_{\infty}(\mathcal{H})$, then $\left\{\lambda_{k}(T)\right\}$ is the sequence of its (nonzero) eigenvalues, numbered with algebraic multiplicity taken into account. If also $T=T^{*}$, then $\lambda_{k}^{(+)}(T)$ is the nonincreasing sequence of positive eigenvalues of $T$, and $\lambda_{k}^{(-)}(T):=\lambda_{k}^{(+)}(-T)$. Next, $n_{ \pm}(s, T)=\operatorname{card}\left\{k \mid \lambda_{k}^{( \pm)}(T)>s\right\}, s>0$. Denote by $\mathbf{S}_{\mathbf{p}}(\mathcal{H}), 1 \leq p<\infty$, the normed ideal of operators $T \in \mathbf{S}_{\infty}(\mathcal{H})$, with

$$
\|T\|_{p}^{p}:=\sum_{k}\left(\lambda_{k}\left(T^{*} T\right)\right)^{p / 2}<\infty
$$

In particular, $\mathbf{S}_{\mathbf{1}}(\mathcal{H})$ is the class of trace-class operators, and $\mathbf{S}_{\mathbf{2}}(\mathcal{H})$ the class of HilbertSchmidt operators.

Below, $H_{0}$ and $H$ are a basic pair of selfadjoint operators acting in $\mathcal{H}$. For resolvents and spectral measures we use the abbreviated notation $R_{z}^{0}=R_{z}\left(H_{0}\right), R_{z}=R_{z}(H)$ and $E_{0}(\cdot)$, $E(\cdot)$, respectively. The condition

$$
V:=H-H_{0} \in \mathbf{S}_{\mathbf{1}}(\mathcal{H})
$$

is always supposed to hold true.

\section{Formulation of the problem. "Naive representation" for the SSF (I. M. Lifshits [1])}

The $\operatorname{SSF} \xi(\lambda)=\xi\left(\lambda ; H, H_{0}\right)$ is determined by the trace formula which was found in the initial paper [1]. It has the form

$$
\operatorname{Tr}\left(\varphi(H)-\varphi\left(H_{0}\right)\right)=\int_{\mathbf{R}} \varphi^{\prime}(\lambda) \xi(\lambda) d \lambda
$$


and is valid for a wide class of functions $\varphi$. The problem of calculating the SSF $\xi$ which enters (1.1) was also first proposed in [1]. Some "explicit" formulas for $\xi$ were obtained there in the case rank $V<\infty$. They follow from the general M. G. Kreĭn's formula (2.3) and so we do not discuss them here. The question of existence of the function $\xi$ satisfying (1.1), of its properties and of the class of admissible functions $\varphi$ was not, of course, discussed in [1]; this was done in [2] under the more general condition (0).

However, the following has been pointed out in [1]. Taking formally $\varphi=\chi_{\mu}$ in (1.1), where $\chi_{\mu}$ is the characteristic function of $\delta_{\mu}=(-\infty, \mu)$, one gets

$$
\xi(\mu)=\operatorname{Tr}\left(E_{0}\left(\delta_{\mu}\right)-E\left(\delta_{\mu}\right)\right) .
$$

This is the representation for the SSF which we call "naive". Unfortunately, generally speaking, (1.2) cannot be understood directly. In [2] an example was described where rank $V=1$, but the corresponding operator

$$
E_{0}\left(\delta_{\mu}\right)-E\left(\delta_{\mu}\right)
$$

is not trace-class and not even Hilbert-Schmidt when $\mu$ is on the spectrum. Thus, the righthand side of (1.2) is ill-defined. At the same time, the trace of the operator (1.3) in the example of [2] can be calculated as the trace of an integral operator, and in this case (1.2) gives the correct expression for $\xi$.

The "naive" formula (1.2) enables one to interpret $\xi$ as the "integrated density of the variation of number of states" under the perturbation. In section 3 we shall explain what kind of precise meaning can be assigned to (1.2).

\section{Representation for the SSF via the perturbation de- terminant (M. G. Kreĭn $[2,3]$ )}

In this approach, the starting object is the perturbation determinant for the pair $H, H_{0}$ which obey the condition $(0)$,

$$
\Delta(z)=\Delta_{H / H_{0}}(z)=\operatorname{det}\left(I+R_{z}^{0} V\right)=\operatorname{det}(H-z I)\left(H_{0}-z I\right)^{-1}, \quad z \in \rho\left(H_{0}\right) .
$$

Consider the logarithm

$$
g(z):=\log \Delta(z)
$$

where the branch is fixed by the condition

$$
g(z) \rightarrow 0, \quad|\operatorname{Im} z| \rightarrow 0
$$

It was shown in [2] that the representation

$$
g(z)=\int_{\mathbf{R}} \frac{\xi(t)}{t-z} d t, \quad \operatorname{Im} z \neq 0
$$

holds true, where $\xi=\bar{\xi} \in L_{1}(\mathbf{R})$. By the inversion formula,

$$
\pi \xi(\lambda)=\lim _{\varepsilon \rightarrow+0} \arg \Delta(\lambda+i \varepsilon), \quad \text { a.e. } \lambda \in \mathbf{R} .
$$


This is the basic Kreı̆n's formula. He proved that the function (2.3) is the SSF. In other words, (1.1) holds true with $\xi$ from (2.3) - at least, for any $\varphi \in C_{l o c}^{1}$ such that

$$
\varphi^{\prime}(\lambda)=\int_{\mathbf{R}} e^{-i \lambda t} \sigma(d t)
$$

where $\sigma$ is a finite complex-valued Borel measure on $\mathbf{R}$. At the same time, the inclusion

$$
\varphi(H)-\varphi\left(H_{0}\right) \in \mathbf{S}_{\mathbf{1}}(\mathcal{H})
$$

was established for such $\varphi$.

The representation (2.3) implies some "natural" properties of the SSF. In particular, the following relations hold true

$$
\begin{gathered}
\int_{\mathbf{R}}|\xi(t)| d t \leq\|V\|_{1}, \\
\int_{\mathbf{R}} \xi(t) d t=\operatorname{Tr} V .
\end{gathered}
$$

The SSF is $L_{1}$-continuous with respect to variations of $V$ in the trace-norm. If $\operatorname{rank} V<\infty$ and $V$ has $p$ positive and $q$ negative eigenvalues, then

$$
-q \leq \xi(t) \leq p, \quad \text { a.e. } t \in \mathbf{R} .
$$

Next, if for some interval $\delta=(a, b)$ and $\varepsilon>0$ one has rank $E_{0}(a-\varepsilon, b+\varepsilon)<\infty$, then

$$
\xi(b-0)-\xi(a+0)=\operatorname{rank} E_{0}(a, b)-\operatorname{rank} E(a, b) .
$$

The relation (2.3) also implies the additivity of the SSF with respect to a successive (additive) introduction of perturbations.

Note that (2.4) holds for $\varphi(\lambda)=\log (\lambda-z)$. Hence, (1.1) and (2.2) imply

$$
\log \Delta_{H / H_{0}}(z)=\operatorname{Tr}\left(\log (H-z I)-\log \left(H_{0}-z I\right)\right), \quad \operatorname{Im} z>0 .
$$

This equality clarifies the role of the perturbation determinant in the derivation of the trace formula (1.1) and the representation (2.3) for the SSF.

The condition (2.4) is far from being necessary for the validity of (2.5) and (1.1). The details on this subject are given in [9], §4.

\section{3 "Naive representation" revisited}

M. G. Kreln's result on the absolute summability of the SSF enables one to put a precise interpretation on the "naive" formula (1.2).

Let $\zeta \in C^{\infty}(\mathbf{R}), 0 \leq \zeta \leq 1, \zeta(x)=1$ for $x \leq-1, \zeta(x)=0$ for $x \geq 0$, and let $\zeta_{\varepsilon}(x):=\zeta(x / \varepsilon), \varepsilon>0$. Take $\varphi(\lambda)=\zeta_{\varepsilon}(\lambda-\mu)$ in (1.1). Obviously, $\varphi$ satisfies the condition (2.4). This implies (2.5) and therefore (1.1) makes sense. The r.h.s. of (1.1) is a convolution $\xi *\left(-\omega_{\varepsilon}\right)$ where $\omega_{\varepsilon}(\lambda)=-\varepsilon^{-1} \zeta^{\prime}(-\lambda / \varepsilon)$ is an approximate identity. Since $\xi \in L_{1}(\mathbf{R})$, the 
convolution $\xi *\left(-\omega_{\varepsilon}\right)$ converges to $-\xi(\mu)$ as $\varepsilon \rightarrow+0$ both in $L_{1}(\mathbf{R})$ and for a.e. $\mu \in \mathbf{R}$. Thus,

$$
\xi(\mu)=\lim _{\varepsilon \rightarrow+0} \operatorname{Tr}\left(\zeta_{\varepsilon}\left(H_{0}-\mu\right)-\zeta_{\varepsilon}(H-\mu)\right), \quad \text { a.e. } \mu \in \mathbf{R} .
$$

Obviously, the operator

$$
\zeta_{\varepsilon}\left(H_{0}-\mu\right)-\zeta_{\varepsilon}(H-\mu)
$$

converges strongly to the operator (1.3) for all $\mu \in \mathbf{R}$. This allows one to accept (3.1) as a precise interpretation of (1.2).

Besides, for $\mu \in \rho\left(H_{0}\right) \cap \rho(H) \cap \mathbf{R}$ the operator (3.2) coincides with (1.3) for small enough $\varepsilon$; in this case (1.2) can be understood straightforwardly.

\section{SSF as a scattering phase (M. Sh. Birman- M. G. Kreйn $[6,7]$ )}

The connection between the SSF and the scattering matrix is deep and far from being apparent. Let us begin with definitions. Let $P_{0}$ (resp. $P$ ) be the projection onto the absolutely continuous subspace of $H_{0}$ (resp. $H$ ). Under the condition (0) the wave operators

$$
W_{ \pm}:=\mathrm{s}-\lim _{t \rightarrow \pm \infty} \exp (i t H) \exp \left(-i t H_{0}\right) P_{0}
$$

exist. (The existence of the limits (4.1) is the essence of the classical theorem of Rosenblum and Kato, see, e.g., [10]). The operator $S=W_{+}^{*} W_{-}$(the scattering operator) is unitary in $P_{0} \mathcal{H}$ and commutes with $H_{0}$. Let $P_{0} \mathcal{H}$ be decomposed into a direct integral

$$
P_{0} \mathcal{H}=\int_{\mathbf{R}} \oplus \mathcal{H}(\lambda) d \lambda
$$

where $H_{0}$ acts as multiplication by $\lambda$. Then $S$ in the decomposition (4.2) acts as multiplication by the operator-valued function $S(\lambda)$, which is called the scattering matrix. The operators $S(\lambda)$ are unitary in $\mathcal{H}(\lambda)$ and

$$
S(\lambda)-I_{\lambda} \in \mathbf{S}_{\mathbf{1}}(\mathcal{H}(\lambda)), \quad \text { a.e. } \lambda \in \mathbf{R} .
$$

The connection between the SSF and the scattering matrix is given by

$$
\operatorname{det} S(\lambda)=\exp (-2 \pi i \xi(\lambda)), \quad \text { a.e. } \lambda \in \mathbf{R}
$$

where $\operatorname{det} S(\lambda)$ makes sense by (4.3).

The relation (4.4) can be considered as one more representation for the SSF. Moreover, (4.4) is sometimes regarded as the definition of $\xi$, which is then called the scattering phase. It is notable that the scattering matrix is related only to absolutely continuous parts of $H_{0}, H$, while the SSF depends on the "full" pair $H_{0}, H$. This is reflected in the fact that the SSF $\xi$ can be determined from (4.4) only up to a singular term which takes integer values almost everywhere. However, in "smooth" situations such a term either does not occur, or can be easily controlled. 
The fundamental role of (4.4) can be explained as follows. Under the condition (0) the operators $H_{0} P_{0}$ and $H P$ are unitarily equivalent. The operators $W_{ \pm}$describe perturbations of the system of eigenfunctions of absolutely continuous spectrum for a fixed $\lambda$. Thus, the scattering theory deals with the "rotation of eigenvectors" for a fixed "eigenvalue". The scattering matrix is one of the characteristics of this "rotation". At the same time, the SSF on the absolutely continuous spectrum describes virtual (infinitesimal) shifts of "eigenvalues"; this better corresponds to the conventional idea of the perturbation theory. Thus, (4.4) connects two different points of view on the spectral essence of the scattering theory.

The relation (4.4) was used (see [6], [7], [10]) to study the spectral properties of the scattering matrix and to obtain estimates for it. In particular, along with (4.3), the following estimate was established:

$$
\int_{\mathbf{R}}\left\|S(\lambda)-I_{\lambda}\right\|_{1} d \lambda \leq 2 \pi\|V\|_{1} .
$$

Later on, stronger and more straightforward methods were found for the investigation of the scattering matrix. But it was the formula (4.4) that first stimulated development in this direction.

In some problems in potential scattering the connection between the $\xi$-function and characteristics of scattering was noticed before the discovery of the general formula (4.4). For example, in the problem on the semiaxis the SSF was expressed in terms of the limit phase (scattering phase) - see [16] as well as [17], where the first rigorous result was obtained. In the three-dimensional scattering problem in the paper [18] the following relation was obtained

$$
\operatorname{Tr} S^{*}(\lambda) \frac{d S(\lambda)}{d \lambda}=2 i \operatorname{Im} \operatorname{Tr}\left(R_{\lambda+i 0}-R_{\lambda+i 0}^{0}\right)
$$

which is close to (4.4). These observations stimulated the search for the abstract formula (4.4). More details can be found in [9], §9.

\section{Representation for the SSF via the integral over the coupling constant (M. Sh. Birman - M. Z. Solomyak $[15])$}

Let $H_{s}=H_{0}+s V, s \in \mathbf{R}$, and $H=H_{1}$. Let $E_{s}(\cdot)$ be the spectral measure for $H_{s}$. Then for any interval $\delta=(a, b)$

$$
\int_{\delta} \xi(\lambda) d \lambda=\int_{0}^{1} \operatorname{Tr}\left(E_{s}(\delta) V\right) d s
$$

Obviously, (5.1) implies

$$
\xi(\lambda)=\frac{d}{d \lambda} \int_{0}^{1} \operatorname{Tr}\left(E_{s}((a, \lambda)) V\right) d s, \quad \text { a.e. } \lambda \in \mathbf{R} .
$$

Formula (5.1) is convenient for producing estimates for the SSF. For example, the following inequalities are valid

$$
\operatorname{Tr} E\left(\delta_{\mu}\right) V \leq \int_{-\infty}^{\mu} \xi(t) d t \leq \operatorname{Tr} E_{0}\left(\delta_{\mu}\right) V, \quad \delta_{\mu}=(-\infty, \mu) .
$$


Both inequalities transfer into (2.6) as $\mu \rightarrow \infty$. Note that $V$ is not supposed to be of definite sign. In its turn, (5.3) implies other useful inequalities. For example, for semibounded from below $H_{0}, H$

$$
\operatorname{Tr} e^{-\beta H} V \leq \beta^{-1} \operatorname{Tr}\left(e^{-\beta H_{0}}-e^{-\beta H}\right) \leq \operatorname{Tr} e^{-\beta H_{0}} V, \quad \beta>0 .
$$

The formula (5.1) was obtained in [15] on the basis of the mechanism of double operator integrals. The main aim was to find a proof of the trace formula which would not rely on complex analytic facts. This aim has not been fully achieved, for the authors failed to obtain an independent proof of the absolute continuity of the measure in the r.h.s. of (5.1). The relations (5.1), (5.2) were the by-products of this attempt. Recently B. Simon [14] has given a more straightforward proof of (5.1).

\section{Representation for the SSF via the integral of the counting function (A. B. Pushnitski [19])}

Here we assume $V \geq 0$ and use the notation $H_{ \pm}=H_{0} \pm V$. As above, we suppose $V \in \mathbf{S}_{1}$. Consider the selfadjoint operators

$$
A(z)=\operatorname{Re} V^{1 / 2} R_{z}^{0} V^{1 / 2}, \quad K(z)=\operatorname{Im} V^{1 / 2} R_{z}^{0} V^{1 / 2}, \quad \operatorname{Im} z>0 .
$$

It is well known (see [11] or [10]) that for a.e. $\lambda \in \mathbf{R}$ there exist the limit values

$$
A(\lambda+i 0) \in \mathbf{S}_{\mathbf{2}}(\mathcal{H}), \quad K(\lambda+i 0) \in \mathbf{S}_{\mathbf{1}}(\mathcal{H}) .
$$

(Moreover, $A(\lambda+i 0) \in \mathbf{S}_{\mathbf{p}}(\mathcal{H})$ for any $p>1$ but in general not for $p=1$; see [12]). It turns out that the SSF can be related to the counting function of the family

$$
M(\lambda, t)=A(\lambda+i 0)+t K(\lambda+i 0), \quad t \in \mathbf{R} .
$$

Namely, the following representation is valid

$$
\pi \xi\left(\lambda ; H_{ \pm}, H_{0}\right)= \pm \int_{\mathbf{R}}\left(1+t^{2}\right)^{-1} n_{\mp}(1, M(\lambda, t)) d t .
$$

Convergence of the integral in (6.3) is ensured by the second of the inclusions (6.1). Formula (6.3) does not allow one to see directly the simplest properties of the SSF, for example, the additivity with respect to perturbations. However, this formula is well adapted for the derivation of the estimates for the SSF and for the calculation of its asymptotics in the large coupling constant limit. We cannot discuss this in more detail here.

Now suppose that $\lambda \in \rho\left(H_{0}\right) \cap \rho(H) \cap \mathbf{R}$. Then (6.2) reduces to the operator $V^{1 / 2} R_{\lambda}^{0} V^{1 / 2}$ and does not depend on $t \in \mathbf{R}$, while (6.3) transfers into

$$
\pm \xi\left(\lambda ; H_{ \pm}, H_{0}\right)=n_{\mp}\left(1, V^{1 / 2} R_{\lambda}^{0} V^{1 / 2}\right) .
$$

The equality (6.4) was obtained earlier in [13] straightforwardly. It can be interpreted as follows. Let $H_{ \pm}(s)=H_{0} \pm s V, 0 \leq s \leq 1$. Then the right-hand side of (6.4) coincides with the number of eigenvalues of $H_{ \pm}(s)$ which cross the point $\lambda$ as $s$ grows from 0 to 1 .

One can use (6.3) for general perturbations (without the restriction on the sign of $V$ ) by introducing successively positive and negative parts of $V$. However, no "unified" formula of the (6.3) type has been found by now in this case. 


\section{Conclusion}

Initially, the spectral shift function $\xi$ was defined by (1.1). Its specification (2.7) leads to the basic M. G. Kreĭn's formula (2.3). Besides, the SSF enters formulas (4.4), (5.2), (6.3) which themselves are surprising and whose derivation from (2.3) is far from being apparent. Each of these formulas casts the light on some of the properties of the SSF, leaving in a shadow others, no less important. This has prompted the authors to write the present essay whose title sounds maybe, too emotional.

\section{References}

[1] I. M. Lifshits, On a problem in perturbation theory, Uspekhi Mat. Nauk 7 (1952), no. 1 (47), 171-180. (Russian)

[2] M. G. Kreŭn, On the trace formula in perturbation theory, Mat. Sb. 33 (75) (1953), 597-626. (Russian)

[3] M. G. Kreĭn, On perturbation determinants and the trace formula for unitary and selfadjoint operators, Dokl. Akad. Nauk SSSR 144 (1962), 268-271; English transl. in Soviet Math. Dokl. 3 (1962).

[4] M. G. Kreln, Some new studies in the theory of perturbations of selfadjoint operators, First Math. Summer School (Kanev, 1963), Part I, "Naukova Dumka", Kiev, 1964, pp.103-187; English transl. in M. G. Kreı̆n, Topics in differential and integral equations and operator theory, Birkhäuser, Basel, 1983, pp. 107-172.

[5] M. G. Kreı̆n, On perturbation determinants and trace formula for certain classes of pairs of operators, J. Operator Theory 17 (1987), 129-187; English transl. in Amer. Math. Soc. Transl. (2) 145 (1989).

[6] M. Sh. Birman and M. G. Kreun, On the theory of wave operators and scattering operators, Dokl. Akad. Nauk SSSR 144 (1962), 475-478; English transl. in Soviet Math. Dokl. 3 (1962).

[7] M. Sh. Birman and M. G. KreIn, Some topics of the theory of the wave operators and scattering operators, Outlines Joint Sympos. Partial Differential Equations (Novosibirsk, 1963), Acad. Sci. USSR Siberian Branch, Moscow, 1963, pp. 39-45. (English)

[8] M. G. Kreĭn and V. A. Yavryan, On spectral shift functions arising in perturbations of a positive operator, J. Operator Theory 6 (1981), 155-191. (Russian)

[9] M. Sh. Birman and D. R. Yafaev, The spectral shift function. The work of M. G. Krein and its further development, Algebra i Analiz 4 (1992), no.5, 1-44; English transl. in St. Petersburg Math. J. 4 (1993), no.5.

[10] D. R. Yafaev, Mathematical scattering theory. General theory, Amer. Math. Soc., Providence, RI, 1992.

[11] M. Sh. Birman and S. B. Entina, The stationary approach in abstract scattering theory, Izv. Akad. Nauk SSSR Ser. Mat. 31 (1967), 401-430; English transl. in Math. USSR Izv. 1 (1967). 
[12] S. N. Naboko, Non-tangent boundary values of operator $R$-functions in the half-plane, Algebra i Analiz, 1 (1989), no.5, 197-222; English transl. in Leningrad Math. J., 1 (1990), no.5.

[13] A. V. Sobolev, Efficient bounds for the spectral shift function, Ann. Inst. H.Poincare, Physique theorique, 58, no.1 (1993), 55-83.

[14] B. Simon, Spectral averaging and the Krĕ̌n spectral shift, submitted to Proc. Amer. Math. Soc.

[15] M. Sh. Birman, M. Z. Solomyak, Remarks on the spectral shift function, Zap. Nauchn. Sem. Leningrad. Otdel. Mat. Inst. Steklov. (LOMI) 27 (1972), 33-46; English transl. in J. Soviet Math. 3 (1975), no.4.

[16] I. M. Lifshits, On the problem of scattering of particles by a centrally symmetric field in quantum mechanics, Khar'kov. Gos. Univ. Uchen. Zap. 27 (1948), 105-107. (Russian)

[17] V. S. Buslaev, L. D. Faddeev, On trace formulas for a singular differential SturmLiouville operator, Dokl. Akad. Nauk SSSR 132 (1960), 13-16; English transl. in Soviet Math. Dokl. 1 (1960).

[18] V. S. Buslaev, Trace formulas for the Schrödinger operator in three-dimensional space, Dokl. Akad. Nauk SSSR 143 (1962), 1067-1070; English transl. in Soviet Math. Dokl. 3 (1962).

[19] A. B. Pushnitski, Representation for the spectral shift function for perturbations of a definite sign, Algebra i Analiz (St.-Petersburg Mathematical Journal), to appear.

Department of Physics,

St.-Petersburg State University,

198904 St.-Petersburg, RUSSIA

1991 Mathematics Subject Classification: Primary 47-02, Secondary 47-03, 47A55, 47B25. 